\title{
Doppler estimates of pulmonary vascular resistance to phenotype pulmonary hypertension in heart failure
}

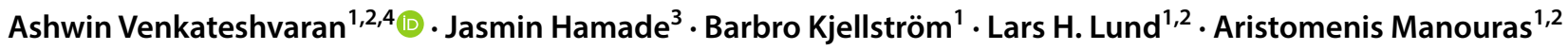

Received: 28 February 2019 / Accepted: 26 March 2019 / Published online: 23 May 2019

(c) The Author(s) 2019

\begin{abstract}
An accurate distinction between isolated post-capillary pulmonary hypertension (Ipc-PH) and combined post- and precapillary pulmonary hypertension $(\mathrm{Cpc}-\mathrm{PH})$ is integral to therapy and prognosis in heart failure (HF). This study aimed to compare the ability of four previously validated Doppler estimates of pulmonary vascular resistance $\left(\mathrm{PVR}_{\text {Doppler }}\right)$ to distinguish Ipc-PH from Cpc-PH in a well-defined HF population. Consecutive subjects referred for HF assessment underwent standard echocardiography immediately followed by right heart catheterization (RHC). Subjects with atrial fibrillation, acute coronary syndrome, significant valvular disease or poor image quality were excluded. $\mathrm{PVR}_{\text {Doppler }}$ estimates were correlated with invasive PVR and agreement was studied using Bland-Altman analysis. Receiver operating characteristics analyses were performed to determine the ability of $\mathrm{PVR}_{\text {Doppler }}$ methods to identify PVR $>3 \mathrm{WU}$. $55 \mathrm{HF}$ subjects (58 \pm 16 years, $55 \%$

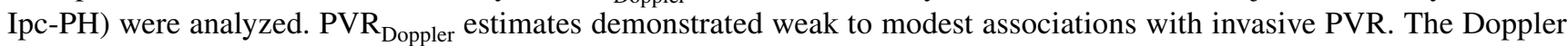
method proposed by Abbas et al. demonstrated relatively strong discriminatory ability to distinguish Ipc-PH from Cpc-PH $(\mathrm{AUC}=0.79 ; 95 \%$ CI 0.63-0.96; $\mathrm{p}=0.001)$. However, Bland-Altman analysis revealed wide limits of agreement $(\mathrm{bias}=0$; $\mathrm{SD}=1.83 \mathrm{WU}$ ) and greater variability at higher mean PVR. Conclusions: $\mathrm{PVR}_{\text {Doppler }}$ estimates demonstrate reasonable ability to distinguish Ipc-PH from Cpc-PH but may not be reliable independent PH distinguishers in HF.
\end{abstract}

Keywords Post-capillary pulmonary hypertension $\cdot$ Doppler echocardiography $\cdot$ Heart failure

\section{Introduction}

Subjects with heart failure (HF) commonly present with post-capillary pulmonary hypertension $(\mathrm{PH})$, which is associated with poor prognosis $[1,2]$. Based on severity of pulmonary vascular resistance (PVR), current guidelines classify the PH into: a) isolated post-capillary PH (Ipc-PH), characterized by pulmonary venous congestion with passive rise in pulmonary pressures and b) combined post- and precapillary $\mathrm{PH}(\mathrm{Cpc}-\mathrm{PH})$ marked by additional superimposed

Ashwin Venkateshvaran

ashwin.venkateshvaran@ki.se

1 Department of Medicine, Karolinska Institutet, Solna, Stockholm, Sweden

2 Heart and Vascular Theme, Karolinska University Hospital, Stockholm, Sweden

3 Department of Clinical Physiology, Karolinska Institutet, Huddinge, Stockholm, Sweden

4 Cardiovascular Research Unit, Norrbacka S1:02, Karolinska University Hospital, 17176 Stockholm, Sweden pulmonary vascular functional and structural alterations [3]. An accurate distinction between these two $\mathrm{PH}$ subgroups is imperative to diagnosis, prognosis and optimal therapy as subjects with $\mathrm{Cpc}-\mathrm{PH}$ demonstrate worse clinical outcome and higher mortality [4].

PVR measurement by right heart catheterization $\left(\mathrm{PVR}_{\mathrm{RHC}}\right)$ is the preferred method to distinguish $\mathrm{PH}$ phenotypes in HF. However, accurate noninvasive PVR estimates are highly desirable to minimize health costs, patient discomfort and exposure to radiation. In subjects with advanced HF, non-invasive assessment of PVR may also be useful in serial assessment of hemodynamic suitability for heart transplantation and for tailoring pharmacological therapy. Multiple methods to assess PVR by Doppler ( $\mathrm{PVR}_{\text {Doppler }}$ ) have been proposed but are not widely utilized owing to inconclusive and conflicting results [5-10]. Further, no studies have specifically compared the discriminatory strength of $\mathrm{PVR}_{\text {Doppler }}$ methods to distinguish Ipc-PH from Cpc-PH in a well-defined HF population.

With the background, we aimed to explore the utility of Doppler methods to distinguish PH phenotypes in HF by 
comparing four validated $\mathrm{PVR}_{\text {Doppler }}$ methods with reference standard $\mathrm{PVR}_{\mathrm{RHC}}$.

\section{Methods}

\section{Patient population}

All consecutive subjects referred to the Karolinska University Hospital for right heart catheterization (RHC) from February 2014 through February 2018 for the assessment of HF were screened for enrollment. All subjects were hemodynamically stable during assessment and medical therapy was suitably titrated. Subjects with acute coronary syndrome or cardiac surgery within a period of $<1$ year prior to RHC, in atrial fibrillation or under pacemaker therapy, with significant concomitant valvular disease or poor image quality were excluded. The study was approved by the local ethics committee (DNR 2008/1695-31) and all patients provided written informed consent.

\section{Echocardiography}

All patients underwent a standard echocardiogram in keeping with current guidelines [11] employing a Vivid E9 ultrasound system (GE Ultrasound, Horten. Norway) equipped with a $2.5 \mathrm{MHz}$ matrix array transducer. 2D gray-scale images were acquired at 50-80 frames/s over three heart cycles. Doppler tracings were recorded using a sweep speed of $100 \mathrm{~mm} / \mathrm{s}$. All images were subsequently exported and analyzed offline (EchoPAC PC, version 11.0.0.0 GE Ultrasound, Waukesha, Wisconsin) by experienced operators blinded to catheterization data. Left ventricular ejection fraction (LVEF) was estimated using the Simpson's biplane

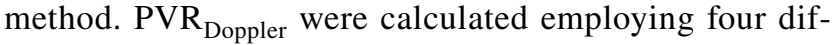
ferent methods as previously described [5-8]. To estimate $\mathrm{PVR}_{\text {Doppler }}$ in the two methods proposed by Abbas et al. [5, $6]$, peak tricuspid regurgitation velocity $\left(\mathrm{TRV}_{\max }\right)$ was measured considering the most optimal signal obtained using apical and parasternal windows and right ventricular outflow tract velocity time index $\left(\right.$ RVOT $\left._{\mathrm{VTI}}\right)$ was obtained by placing a $5 \mathrm{~mm}$ pulse-wave (PW) Doppler sample volume proximal to the pulmonary valve in the right ventricular outflow tract (RVOT) and tracing the resultant spectral wave form. For the method proposed by Scapellato et al. [7], pre-ejection period (PEP) was defined as time between QRS-start and RVOT-onset. Acceleration time (AcT) was expressed as the time between RVOT-onset and the RVOT-peak while total time (TT) was expressed as time interval between RVOTonset and the RVOT-end. Finally, to estimate PVR using the method proposed by Haddad et al. RVOT ${ }_{\mathrm{VTI}}$ was measured as previously described, systolic pulmonary artery pressure (SPAP) was obtained by adding the recommended estimates of RA pressure [11] to the corresponding pressure gradient obtained from the $\mathrm{TRV}_{\max }$ signal.

\section{Right heart catheterization}

RHC was performed using a 6F Swan Ganz catheter employing jugular or femoral vein access within $1 \mathrm{~h}$ post echocardiographic evaluation. Mean right atrial pressure $\left(\mathrm{RAP}_{\mathrm{M}}\right)$, systolic-, diastolic- and mean pulmonary artery pressure $\left(\mathrm{PAP}_{\mathrm{S}}, \mathrm{PAP}_{\mathrm{D}}, \mathrm{PAP}_{\mathrm{M}}\right)$ and mean pulmonary arterial wedge pressure $\left(\mathrm{PAWP}_{\mathrm{M}}\right)$ were obtained under fluoroscopic guidance after calibration with the zero-level set at the midthoracic line. Oxygen consumption was measured breathby-breath by dedicated gas analysis system. Cardiac output (CO) was measured using the Fick's principle. PVR $_{\mathrm{RHC}}$ was calculated as $\mathrm{PVR}_{\mathrm{RHC}}=\left(\mathrm{PAP}_{\mathrm{M}}-\mathrm{PAWP}_{\mathrm{M}}\right) / \mathrm{CO}$, transpulmonary gradient $(\mathrm{TPG})$ as $\mathrm{TPG}=\mathrm{PAP}_{\mathrm{M}}-\mathrm{PAWP}_{\mathrm{M}}$ and diastolic pulmonary gradient $(\mathrm{DPG})$ as $\mathrm{DPG}=\mathrm{PAP}_{\mathrm{D}}-\mathrm{PAWP}_{\mathrm{M}}$. Postcapillary $\mathrm{PH}$ was defined as RHC derived $\mathrm{PAP}_{\mathrm{M}} \geq 25 \mathrm{mmHg}$ and $\mathrm{PAWP}_{\mathrm{M}}>15 \mathrm{mmHg}$. Patients were further divided into Ipc-PH (PVR $\leq 3$ and/or DPG $<7$ ) and Cpc-PH (PVR $>3$ and/or DPG $\geq 7$ ) in keeping with current recommendations [3]. All pressure tracings were stored (WITT Series III, Witt Biomedical Corp., Melbourne, FL) and analyzed off-line by one experienced operator.

\section{Statistical methods}

Normality was tested using the Shapiro-Wilk test and visually reaffirmed using QQ plots. Continuous variables are expressed as mean $\pm \mathrm{SD}$ or median and interquartile range. Categorical variables are expressed as numbers and percentage. Ipc-PH and Cpc-PH subgroups were compared using two-sample Student's or Man-Whitney test. Correlations between echocardiographic and invasive measurements were performed using the Pearson's 2-tailed test. Multivariate regression analysis was performed to study association

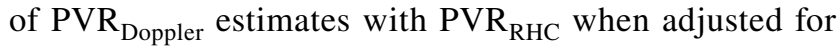
confounders. Receiver operator characteristics (ROC) analysis was performed to determine the discriminatory ability of the selected Doppler method to identify elevated PVR (>3WU). Statistical significance of the difference between the areas of multiple ROC curves was calculated using the method of Delong et al. [12]. Regression equations were derived for each of the Doppler-derived PVR methods and agreement between echocardiographic and invasive PVR was showcased using Bland-Altman plots. Tests were performed at $95 \%$ confidence intervals and a $p$ value $\leq 0.05$ was considered statistically significant. To evaluate the intra- and inter-observer variability of the methods, ten subjects were measured twice by the same investigator and by two different investigators and interclass correlation coefficient for absolute agreement and mean value and standard deviation 
of differences were calculated. All methods demonstrated adequate to good feasibility and excellent intra-observer and inter-observer concordances (Table 1). IBM SPSS statistics version 23.0 was employed for analysis.

\section{Results}

Of 210 subjects referred for RHC during the study period, 55 fulfilled criteria and were included in the analysis (Fig. 1). Patient characteristics are presented in Table 2. All subjects demonstrated signs and symptoms of HF, elevated NTproBNP and objective evidence of ventricular systolic and/or diastolic dysfunction. Thirty-two subjects (58\%) were classified as Ipc-PH and 23 (42\%) as Cpc-PH (Table 2). More than half the subjects demonstrated a LVEF $<50 \%$ $(\mathrm{n}=30 ; 54 \%)$.

Patients with Cpc-PH were older, more often female (Table 2) and had higher PA pressures, PVR and lower CO (Table 3) than patients with Ipc-PH. Echocardiographic evaluation revealed no differences in LVEF or ventricular dimensions between the two groups. PVR echo surrogates proposed by Abbas et al. [5, 6]. and Haddad et al. [8]. were higher in Cpc-PH than in Ipc-PH while the method proposed by Scapellato et al. [7]. showed no difference between groups (Table 3). Eight subjects (14\%) demonstrated no or
Table 1 Feasibility and reproducibility of Dopplerbased PVR methods

Fig. 1 Patient flow chart

\begin{tabular}{llllll}
\hline PVR $_{\text {Doppler }}$ methods & Feasibility (\%) & $\begin{array}{l}\text { Inter-class cor- } \\
\text { relation }\end{array}$ & & $\begin{array}{l}\text { Intra-class cor- CI } \\
\text { relation }\end{array}$ \\
\hline Abbas et al. [5] & 86 & 0.98 & $0.95-0.99$ & 0.99 & $0.97-0.99$ \\
Abbas et al. [6] & 86 & 0.98 & $0.96-0.99$ & 0.99 & $0.98-0.99$ \\
Scapellato et al. [7] & 93 & 0.93 & $0.90-0.95$ & 0.95 & $0.93-0.97$ \\
Haddad et al. [8] & 86 & 0.98 & $0.94-0.99$ & 0.99 & $0.97-0.99$ \\
\hline
\end{tabular}


Table 2 Characteristics of patient population

\begin{tabular}{|c|c|c|c|c|}
\hline & All $(\mathrm{n}=55)$ & Ipc-PH $(n=32)$ & Cpc-PH (n=23) & $\mathrm{p}$-Value \\
\hline \multicolumn{5}{|l|}{ Demographics } \\
\hline Age (years) & $58 \pm 16$ & $54 \pm 15$ & $63 \pm 17$ & $\mathbf{0 . 0 3}$ \\
\hline Female & $18(33)$ & $5(15)$ & $13(56)$ & $<0.001$ \\
\hline \multicolumn{5}{|l|}{ Medical history } \\
\hline Diabetes & $15(27)$ & $10(31)$ & $5(21)$ & 0.48 \\
\hline Hypertension & $30(54)$ & $17(53)$ & $13(56)$ & 0.68 \\
\hline Hypercholesteremia & $17(30)$ & $7(21)$ & $10(43)$ & 0.18 \\
\hline \multicolumn{5}{|l|}{ NYHA class } \\
\hline I/II/III/IV & $2 / 7 / 42 / 4(4 / 13 / 76 / 7)$ & $2 / 6 / 21 / 3(6 / 18 / 65 / 9)$ & $0 / 1 / 21 / 1(0 / 4 / 92 / 4)$ & 0.17 \\
\hline \multicolumn{5}{|l|}{ Clinical assessment } \\
\hline HR (bpm) & $67(60 ; 82)$ & $66(60 ; 79)$ & $70(60 ; 88)$ & 0.43 \\
\hline $\operatorname{BSA}\left(m^{2}\right)$ & $1.9 \pm 0.2$ & $2.0 \pm 0.2$ & $1.9 \pm 0.2$ & 0.02 \\
\hline $\mathrm{SBP}(\mathrm{mmHg})$ & $112 \pm 26$ & $111 \pm 26$ & $114 \pm 26$ & 0.67 \\
\hline $\mathrm{DBP}(\mathrm{mmHg})$ & $64 \pm 14$ & $62 \pm 12$ & $68 \pm 17$ & 0.19 \\
\hline \multicolumn{5}{|l|}{ Laboratory } \\
\hline NTproBNP (ng/L) & $2480(1310 ; 3110)$ & $2480(1310 ; 2930)$ & $2385(855 ; 3507)$ & 0.95 \\
\hline $\mathrm{Hb}(\mathrm{g} / \mathrm{L})$ & $129 \pm 21$ & $132 \pm 22$ & $124 \pm 18$ & 0.16 \\
\hline Serum creatinine $(\mu \mathrm{mol} / \mathrm{L})$ & $105(85 ; 130)$ & $106(85 ; 130)$ & $105(84 ; 130)$ & 0.62 \\
\hline \multicolumn{5}{|l|}{ Diagnosis } \\
\hline $\mathrm{RCM}$ & $6(11)$ & $4(13)$ & $2(9)$ & \\
\hline DCM & $6(11)$ & $5(16)$ & $1(4)$ & \\
\hline $\mathrm{HCM}$ & $4(7)$ & $3(9)$ & $1(4)$ & \\
\hline ICM & $20(36)$ & $9(28)$ & $11(48)$ & \\
\hline Multifactorial & $18(33)$ & $10(31)$ & $8(35)$ & \\
\hline Myocarditis & $1(2)$ & $1(3)$ & $0(0)$ & \\
\hline
\end{tabular}

Bold text was chosen to emphasize that these values were significant $(p<0.05)$

Data presented as mean $\pm \mathrm{SD} /$ median $(\mathrm{Q} 1 ; \mathrm{Q} 3)$ or number $(\%)$. P-value represents difference between Ipc$\mathrm{PH}$ and $\mathrm{Cpc}-\mathrm{PH}$

$I p c-P H$ isolated post-capillary pulmonary hypertension $(\mathrm{PH}), C p c-P H$ combined post- and pre-capillary, $N Y H A$ New York heart association function class, $H R$ heart rate, $B S A$ body surface area, SBP systolic blood pressure, $D B P$ diastolic blood pressure, $N T$ proBNP N-terminal pro b-type natriuretic peptide, $H b$ hemoglobin, $R C M$ restrictive cardiomyopathy, $D C M$ dilated cardiomyopathy, $H C M$ hypertrophic cardiomyopathy, ICM ischemic cardiomyopathy insignificant TR, 27 subjects (50\%) demonstrated mild TR, $17(31 \%)$ moderate and $3(5 \%)$ had severe TR.

\section{Association between echocardiographic and invasive PVR estimates}

PVR $_{\text {Doppler }}$ methods proposed Abbas et al. [5, 6] and Haddad et al. [8]. demonstrated significant associations with invasive PVR. Of the four methods, The revised method proposed by Abbas et al. [6]. demonstrated the strongest association with invasive PVR (Table 4) and was an independent predictor of invasive PVR as per the regression equation $\mathrm{PVR}_{\text {Abbas }}=1.31+2.59 \mathrm{x}\left[(\mathrm{TRV} \max )^{2} / \mathrm{VTI}\right]$. Even when adjusted for age, gender and BMI, this method continued to demonstrate strongest association with $\operatorname{PVR}_{\mathrm{RHC}}(\beta=0.63$, $\mathrm{p}<0.001)$. No significant correlations were observed between Doppler-derived and invasive PVR when Cpc-PH patients were separately analyzed $(\mathrm{p}>0.05)$. Comparison between echocardiographic and corresponding RHC variables demonstrated a weak association between RVOT $_{\mathrm{VTI}}$ and Fick-derived $\mathrm{CO}(\mathrm{r}=0.38 ; \mathrm{p}<0.01)$ and modest relationship between $\mathrm{TRV}_{\text {max }}$ and invasive $\operatorname{PAP}_{\mathrm{s}}(\mathrm{r}=0.52 ; \mathrm{p}<0.001)$.

\section{PVR $_{\text {Doppler }}$ ability to distinguish PH phenotypes}

PVR $_{\text {Doppler }}$ by methods proposed by Abbas et al. [5, 6] and Haddad et al. [8] demonstrated a fair discriminatory ability to distinguish Ipc-PH from $\mathrm{Cpc}-\mathrm{PH}$ subjects in our patient group (Table 5). Of these three, the revised method proposed by Abbas et al. [6] demonstrated highest area under the curve (AUC $=0.79 ; 95 \%$ CI 0.63-0.96; $\mathrm{p}=0.001$ ) (Table 5 and Fig. 2a). A cut-off value of 0.59 provided best balanced sensitivity $(81 \%)$ and specificity $(65 \%)$ to determine PVR > 3WU. Bland-Altman analysis, however, revealed 
Table 3 Invasive and echocardiographic data of patient population

$$
\text { All post-capillary } \mathrm{PH}(\mathrm{n}=55)
$$

Ipc-PH $(\mathrm{n}=32 ; \mathrm{PVR} \leq 3$ and/ or DPG $<7)$

Right heart catheterization

\begin{tabular}{|c|c|c|c|c|}
\hline $\mathrm{PAWP}_{\mathrm{M}}(\mathrm{mmHg})$ & $24 \pm 6$ & $24 \pm 6$ & $25 \pm 6$ & 0.98 \\
\hline $\mathrm{PAP}_{\mathrm{S}}(\mathrm{mmHg})$ & $58 \pm 15$ & $52 \pm 12$ & $66 \pm 15$ & $<0.001$ \\
\hline $\mathrm{PAP}_{\mathrm{D}}(\mathrm{mmHg})$ & $24 \pm 7$ & $23 \pm 6$ & $26 \pm 8$ & 0.04 \\
\hline $\mathrm{PAP}_{\mathrm{M}}(\mathrm{mmHg})$ & $38 \pm 9$ & $34 \pm 7$ & $43 \pm 10$ & 0.001 \\
\hline RAP (mmHg) & $12 \pm 6$ & $12 \pm 6$ & $12 \pm 6$ & 0.98 \\
\hline PVR (WU) & $3.3 \pm 2.1$ & $2.0 \pm 0.7$ & $5.2 \pm 2.0$ & $<0.001$ \\
\hline TPG (mmHg) & $13 \pm 6$ & $10 \pm 3$ & $18 \pm 6$ & $<0.001$ \\
\hline $\mathrm{CO}(\mathrm{L} / \mathrm{min})$ & $4.4 \pm 1.5$ & $5.1 \pm 1.6$ & $3.6 \pm 0.9$ & $<0.001$ \\
\hline \multicolumn{5}{|l|}{ Echocardiography } \\
\hline LVIDd (mm) & $57 \pm 13$ & $58 \pm 11$ & $54 \pm 14$ & 0.28 \\
\hline LVEF (\%) & $44 \pm 22$ & $40 \pm 19$ & $46 \pm 18$ & 0.27 \\
\hline LVEF < 50\% (n) & $30(54)$ & $19(34)$ & $11(20)$ & 0.32 \\
\hline RVIDd (mm) & $43 \pm 8$ & $43 \pm 8$ & $43 \pm 7$ & 0.88 \\
\hline RA Area $\left(\mathrm{cm}^{2}\right)$ & $22 \pm 8$ & $16 \pm 6$ & $22 \pm 9$ & 0.65 \\
\hline TAPSE (mm) & $16 \pm 6$ & $16 \pm 6$ & $16 \pm 6$ & 0.73 \\
\hline $\mathrm{TRV}_{\max }(\mathrm{m} / \mathrm{s})$ & $3.1 \pm 0.7$ & $3.0 \pm 0.6$ & $3.3 \pm 0.9$ & 0.002 \\
\hline RVSP (mmHg) & $56 \pm 17$ & $49 \pm 12$ & $64 \pm 21$ & 0.002 \\
\hline E/e'septal & $17 \pm 9$ & $17 \pm 9$ & $18 \pm 9$ & 0.74 \\
\hline $\operatorname{LAVI}\left(\mathrm{ml} / \mathrm{m}^{2}\right)$ & $54 \pm 14$ & $54 \pm 15$ & $55 \pm 16$ & 0.86 \\
\hline \multicolumn{5}{|l|}{ PVR $_{\text {Doppler }}$ methods } \\
\hline Abbas et al. [5] & $0.22 \pm 0.11999 .00$ & $0.18 \pm 0.08$ & $0.30 \pm 0.13$ & $<0.001$ \\
\hline Abbas et al. [6] & $0.75 \pm 0.48$ & $0.56 \pm 0.28$ & $1.00 \pm 0.6$ & $<0.001$ \\
\hline Scapellato et al. [7] & $0.003 \pm 0.001$ & $0.003 \pm 0.001$ & $0.004 \pm 0.002$ & 0.13 \\
\hline Haddad et al. [8] & $0.05 \pm 0.03$ & $0.04 \pm 0.02$ & $0.08 \pm 0.04$ & 0.01 \\
\hline
\end{tabular}

Bold text was chosen to emphasize that these values were significant $(p<0.05)$

Data presented as mean \pm SD or number (\%). P-value represents difference between Ipc-PH and Cpc-PH

$P H$ pulmonary hypertension, $I p c-P H$ isolated post-capillary pulmonary hypertension, $C p c-P H$ combined post- and pre-capillary pulmonary hypertension, $P A W P_{M}$ mean pulmonary capillary wedge pressure, $P A P$ pulmonary artery pressure, $s$ systolic, $d$ diastolic, $\mathrm{m}$ mean, $R A P$ right atrial pressure, $P V R$ pulmonary vascular resistance, $T P G$ transpulmonary gradient, $C O$ cardiac output, $L V I D d$ left ventricular internal diameter during end-diastole, $E F$ ejection fraction, $R V I D$ right ventricular internal diameter end-diastole, $R A$ right atrium, TAPSE tricuspid annular plane systolic excursion, $T R V_{\max }$ tricuspid regurgitation max velocity, $R V S P$ right ventricular systolic pressure, $E / e^{\prime}$ transmitral early diastolic to myocardial early diastolic velocity ratio, $L A V I$ left atrial volume index, $R V O T_{V T I}$ velocity time integral across right ventricular outflow tract, $P E P$ pre-ejection period, $A c T$ acceleration time, $T T$ total time, $S P A P$ systolic pulmonary artery pressure

Table 4 Correlations between invasive and Doppler-based PVR

\begin{tabular}{lllc}
\hline PVR $_{\text {Doppler }}$ methods & PVR formula & R value & P value \\
\hline Abbas et al. [5] & $\mathrm{TRV}_{\max } / \mathrm{RVOT}_{\text {VTI }}$ & 0.52 & $<0.001$ \\
Abbas et al. [6] & $\mathrm{TRV}_{\max }^{2} / \mathrm{RVOT}_{\text {VTI }}$ & 0.57 & $<0.001$ \\
Scapellato et al. [7] & $(\mathrm{PEP} / \mathrm{AcT}) / \mathrm{TT}$ & 0.37 & 0.06 \\
Haddad et al. [8] & $\mathrm{SPAP} /\left(\mathrm{HR} \times \mathrm{RVOT}_{\mathrm{VTI}}\right)$ & 0.50 & $<0.001$ \\
\hline
\end{tabular}

wide limits of agreement between RHC and Doppler-based PVR (bias $=0 ; \mathrm{SD}=1.83 \mathrm{WU}$ ) and greater variability was observed with higher PVR (Fig. 2b). Further, when the difference between $\mathrm{PVR}_{\text {Doppler }}$ and $\mathrm{PVR}_{\mathrm{RHC}}$ was expressed as percentage and plotted against $\mathrm{PVR}_{\mathrm{RHC}}$, a large relative difference was observed in the setting of non-elevated
PVR (Fig. 2c). In patients with PVR $\leq 3 \mathrm{WU}$, Bland-Altman demonstrated better limits of agreement but greater bias with Doppler-based PVR demonstrating higher values as compared with invasive measurements (Bias $=0.8$; $\mathrm{SD}=0.76 \mathrm{WU})$. No significant difference was observed when comparing AUC curves between PVR ${ }_{\text {Doppler }}$ methods proposed by Abbas et al. [5, 6] and Haddad et al. [8] (p>0.05 for all comparisons).

\section{Discussion}

The ability of Doppler derived methods to assess PVR has been debated. Early studies focused on pulmonary artery morphological flow patterns demonstrated poor association 
Table 5 Discriminatory ability of Doppler-based PVR methods to distinguish Ipc-PH and $\mathrm{Cpc}-\mathrm{PH}$ and Bland-Altman analysis $(\mathrm{n}=55)$

\begin{tabular}{llllll}
\hline PVR $_{\text {Doppler }}$ methods & PVR formula & AUC & CI & P value & $\begin{array}{l}\text { Bland-Altman } \\
\text { mean differ- } \\
\text { ence } \pm \text { SD }\end{array}$ \\
\hline Abbas et al. [5] & TRV $_{\max } / \mathrm{RVOT}_{\text {VTI }}$ & 0.77 & $0.60-0.93$ & 0.003 & $0.02 \pm 1.89$ \\
Abbas et al. [6] & TRV $_{\text {max }}^{2} / \mathrm{RVOT}_{\mathrm{VTI}}$ & 0.79 & $0.63-0.96$ & 0.001 & $0.00 \pm 1.83$ \\
Scapellato et al. [7] & $(\mathrm{PEP} / \mathrm{AcT}) / \mathrm{TT}$ & 0.60 & $0.44-0.76$ & 0.195 & $0.00 \pm 2.01$ \\
Haddad et al. [8] & $\mathrm{SPAP} /\left(\mathrm{HR} \times \mathrm{RVOT}_{\mathrm{VTI}}\right)$ & 0.72 & $0.54-0.86$ & 0.012 & $0.00 \pm 1.91$ \\
\hline
\end{tabular}

with invasive data [13, 14]. More recently, investigations incorporating Doppler surrogates of TPG and CO have exhibited greater potential to accurately represent $\mathrm{PVR}_{\mathrm{RHC}}$ in pulmonary arterial hypertension $[8,10]$ cardiomyopathy $[15,16]$, chronic HF with severe systolic dysfunction [7] and mixed $\mathrm{PH}$ populations $[5,6]$. To our knowledge, a comparison between multiple Doppler assessments to specifically distinguish Ipc-PH from Cpc-PH in the context of HF has not been performed.

Abbas et al. in their first study suggested that the relationship between $\mathrm{TRV}_{\max }$ and $\mathrm{VTI}_{\mathrm{RVOT}}$-noninvasive surrogates of right sided pressure and flow demonstrated a strong correlation and satisfactory limits of agreement with invasively obtained PVR [5]. As compared with that study, our patient group demonstrated more severe PVR, higher PA pressures and lower EF suggesting a more severe clinical presentation. Scapellato and colleagues enrolled subjects with chronic HF in their validation effort [7]. While all their subjects presented with severely impaired LV systolic performance, a closer inspection of the hemodynamic profile of that group reveals less severe degrees of pulmonary vascular derangements as compared with our cohort. The generally weaker associations with invasive measurements exhibited by the aforementioned methods in our cohort may partially be attributed to less severe clinical presentations, and to the inherent limitations of these methods to assess higher PVR [9].

To address these limitations, Haddad et al. [8], and in a revised version, Abbas et al. [6] chose to validate newer Doppler methods aimed at subjects with more severe PVR elevation. While these two methods demonstrated relatively stronger correlation with invasive PVR, the strength of association was modest. Both Haddad et al. [8]. and Abbas et al. [6]. incorporated $\mathrm{TVI}_{\mathrm{RVOT}}$ and $\mathrm{TRV}_{\max }$ in their equations, which demonstrated only modest correlations with corresponding invasive measurements. While the reason for a lack of strong association is unclear, one can speculate that the determination of $\mathrm{TRV}_{\max }$ may be a potential source of error in the specific setting of high PVR. In the simplified Bernoulli's equation, pulmonary artery systolic pressure gradient is estimated by squaring $\mathrm{TRV}_{\max }$. Given this relationship, small changes in $\mathrm{TRV}_{\text {max }}$ will likely reflect larger changes in PA pressures in subjects with more severe $\mathrm{PH}$ as compared with those with no or mild $\mathrm{PH}$, predisposing this method to greater error. The lack of association between Dopplerderived and invasive PVR in the specific context of $\mathrm{Cpc}-\mathrm{PH}$ in our cohort further strengthens this speculation. Further, the absence of measurable TR does not necessarily rule out significant $\mathrm{PH}$ [17], suggesting that overt dependency on this echocardiographic component to determine possible elevations in PVR may not always be reliable.

The identification of Doppler methods that accurately distinguish Ipc-PH from Cpc-PH is particularly attractive in $\mathrm{HF}$, as a surrogate for RHC during screening for HF device therapies and heart transplantation, assessments of pharmacological therapy and to predict outcomes. In the present study, Doppler methods demonstrated reasonable ability to distinguish these two $\mathrm{PH}$ groups but may be not be reliable independent diagnostics. Even the best performing of the four tested Doppler indices [6] demonstrated no significant associations in the setting of elevated PVR, had wide limits of agreement with invasive measurements and greater variability as mean PVR increased. These results suggest that the use of Doppler indices in the absence of RHC, as has been proposed in previous studies [18, 19] might not be advisable. Continued search for an accurate noninvasive measure in the specific context of HF with significant PH is warranted.

Strengths of the study include data acquisition where catheterization was performed within $1 \mathrm{~h}$ after echocardiography, minimizing the probability of significantly altered hemodynamic states. Limitations include the assessment of CO using only the Fick method, while a comparison with the measurement of $\mathrm{CO}$ by thermodilution may have added value. Additionally, the exclusion of subjects owing to insignificant TR signals could have been avoided with administration of agitated saline contrast.

\section{Conclusion}

While Doppler-based assessments of PVR demonstrate reasonable ability to distinguish Ipc-PH from $\mathrm{Cpc}-\mathrm{PH}$, wide limits of agreement with invasive measurements and greater variability at higher PVR preclude the wider utilization of these methods as reliable independent $\mathrm{PH}$ distinguisher in the setting of HF. 
Fig. 2 Diagnostic accuracy of Doppler-derived PVR as per method proposed by Abbas et al. a Receiver-operating characteristic curve. A TRV $\mathrm{Tax}_{\max }^{2}$ $\mathrm{TVI}_{\mathrm{RVOT}}$ cut-off value of 0.59 provided best balanced sensitivity $(81 \%)$ and specificity $(65 \%)$ to determine PVR $>3 \mathrm{WU}$.

b Bland-Altman analysis of PVR obtained by Doppler and invasive PVR. c Percentage difference between Dopplerderived and invasive PVR plotted against invasive PVR
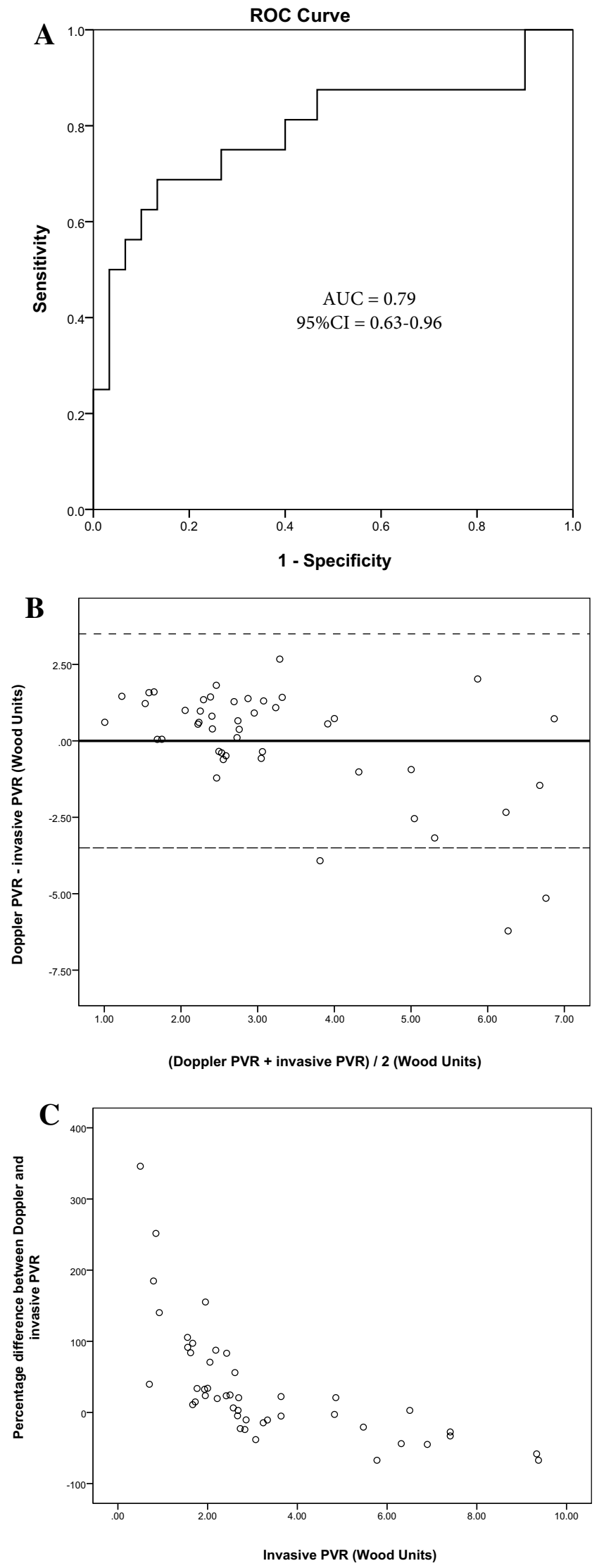
Acknowledgements AV is supported by a grant from the Swedish Association for pulmonary hypertension; LHL is supported by grants from the Swedish Research Council [Grant Nos. 2013-23897-10460423 and 523-2014-2336], Swedish Heart Lung Foundation [Grant Nos. 20100419 and 20120321], Stockholm County Council [Grant Nos. 20110120 and 20140220] and Swedish Society of Medicine [Grant Nos. 174111 and 504881].

\section{Compliance with ethical standards}

\section{Conflict of interest None.}

Open Access This article is distributed under the terms of the Creative Commons Attribution 4.0 International License (http://creativeco mmons.org/licenses/by/4.0/), which permits unrestricted use, distribution, and reproduction in any medium, provided you give appropriate credit to the original author(s) and the source, provide a link to the Creative Commons license, and indicate if changes were made.

\section{References}

1. Vachiery J-L et al (2013) Pulmonary hypertension due to left heart diseases. J Am Coll Cardiol 62(25 Supplement):D100-D108

2. Rosenkranz S et al (2016) Left ventricular heart failure and pulmonary hypertension. Eur Heart J 37(12):942-954

3. Galiè $\mathrm{N}$ et al (2015) 2015 ESC/ERS guidelines for the diagnosis and treatment of pulmonary hypertension: the Joint Task Force for the Diagnosis and Treatment of Pulmonary Hypertension of the European Society of Cardiology (ESC) and the European Respiratory Society (ERS): endorsed by: association for European Paediatric and Congenital Cardiology (AEPC), International Society for Heart and Lung Transplantation (ISHLT). Eur Heart J 37(1):67-119

4. Aronson D et al (2003) The relationship between reactive pulmonary hypertension and mortality in patients with acute decompensated heart failure. Circulation 4:110.960864

5. Abbas AE et al (2003) A simple method for noninvasive estimation of pulmonary vascular resistance. J Am Coll Cardiol 41(6):1021-1027

6. Abbas AE et al (2013) Noninvasive assessment of pulmonary vascular resistance by Doppler echocardiography. J Am Soc Echocardiogr 26(10): 1170-1177

7. Scapellato F et al (2001) Accurate noninvasive estimation of pulmonary vascular resistance by Doppler echocardiography in patients with chronic heart failure. J Am Coll Cardiol 37(7):1813-1819
8. Haddad F et al (2009) A novel non-invasive method of estimating pulmonary vascular resistance in patients with pulmonary arterial hypertension. J Am Soc Echocardiogr 22(5):523-529

9. Rajagopalan $\mathrm{N}$ et al (2009) Noninvasive estimation of pulmonary vascular resistance in pulmonary hypertension. Echocardiography 26(5):489-494

10. Kouzu H et al (2009) Noninvasive estimation of pulmonary vascular resistance by Doppler echocardiography in patients with pulmonary arterial hypertension. Am J Cardiol 103(6):872-876

11. Lang RM et al (2015) Recommendations for cardiac chamber quantification by echocardiography in adults: an update from the American Society of Echocardiography and the European Association of Cardiovascular Imaging. Eur Heart J Cardiovasc Imag 16(3):233-270

12. DeLong ER, DeLong DM, Clarke-Pearson DL (1988) Comparing the areas under two or more correlated receiver operating characteristic curves: a nonparametric approach. Biometrics 44(3):837-845

13. Martin-Duran R et al (1986) Comparison of Doppler-determined elevated pulmonary arterial pressure with pressure measured at cardiac catheterization. Am J Cardiol 57(10):859-863

14. Dabestani A et al (1987) Evaluation of pulmonary artery pressure and resistance by pulsed Doppler echocardiography. Am J Cardiol 59(6):662-668

15. Markush D et al (2018) Noninvasive echocardiographic measures of pulmonary vascular resistance in children and young adults with cardiomyopathy. J Am Soc Echocardiogr 31:807-815

16. Koestenberger M (2018) Accurate Estimation of Elevated Pulmonary Vascular Resistance Using Sophisticated Echocardiographic Variables in Children with Cardiomyopathy. J Am Soc Echocardiogr 31:1067-1068

17. O'Leary JM et al (2018) Lack of a tricuspid regurgitation doppler signal and pulmonary hypertension by invasive measurement. J Am Heart Assoc 7(13):e009362

18. Ulett KB, Marwick TH (2007) Incorporation of pulmonary vascular resistance measurement into standard echocardiography: implications for assessment of pulmonary hypertension. Echocardiography 24(10):1020-1022

19. Pirat B, McCulloch ML, Zoghbi WA (2006) Evaluation of global and regional right ventricular systolic function in patients with pulmonary hypertension using a novel speckle tracking method. Am J Cardiol 98(5):699-704

Publisher's Note Springer Nature remains neutral with regard to jurisdictional claims in published maps and institutional affiliations. 